VOL. 14 (1975), 23-36.

\title{
On certain non-archimedean functions analogous to complex analytic functions
}

\section{Kurt Mahler}

To the memory of J.G. van der Corput

Let $Q_{P}$ and $K$ be the rational p-adic field and an algebraic extension of $Q_{p}$ of finite degree, respectively, and let $I$ and $I_{K}$ be the subsets of $Q_{p}$ and of $K$ consisting of the $p$-adic integers of these fields.

It is known that the continuous functions $f: I \rightarrow Q_{p}$ can be written as

$$
f(x)=\sum_{n=0}^{\infty} a_{n}\left(\begin{array}{l}
x \\
n
\end{array}\right),
$$

where this series converges uniformly on $I$, and that such continuous functions need not be differentiable at any point. We here study continuous functions $F: I_{K} \rightarrow K$ which for all $X$ on $I_{K}$ are the sum of a uniformly convergent series

$$
F(X)=\sum_{n=0}^{\infty} A_{n}\left(\begin{array}{l}
X \\
n
\end{array}\right)
$$

It is proved that such functions $F(X)$ have at every point of $I_{K}$ derivatives of all orders. In the special case when $K$ is totally ramified, they cannot in general be developed into power series that converge everywhere on $I_{K}$, but this is possible when $K$ is not totally ramified.

Received 23 October 1975. 
Let $p$ be a fixed prime, $|a|_{p}$ the p-adic valuation on 2 normed such that

$$
|p|_{p}=p^{-1}
$$

$Q_{p}$ the $p$-adic closure of $Q$, and $I=\left\{a \in Q_{p},|a|_{p} \leq 1\right\}$ the ring of $p$-adic integers. I have proved [1] that if $f: I \rightarrow Q_{p}$ is a continuous function on $I$ and if

$$
a_{n}=\sum_{k=0}^{n}(-1)^{k}\left(\begin{array}{l}
n \\
k
\end{array}\right) f(n-k)
$$

then $\left\{a_{n}\right\}$ is a p-adic null sequence, and

$$
f(x)=\sum_{n=0}^{\infty} a_{n}\left(\begin{array}{l}
x \\
n
\end{array}\right)
$$

uniformly for all $x$ on $I$.

Consider now an algebraic extension field $K$ of finite degree $N$ over $Q_{p}$. The valuation $|a|_{p}$ can be continued to $K$, and $K$ is still complete with respect to this continuation. The set

$$
I_{K}=\left\{A \in K,|A|_{p} \leq 1\right\}
$$

forms a ring, and we call its element the $K$-integers. We can now consider again the continuous functions $F: I_{K} \rightarrow K$, and these can without difficulty be proved to be representable as $N$-fold interpolation series in the $N$ components $x_{1}, \ldots, x_{N}$ of $X$; here $\Omega_{1}, \ldots, \Omega_{N}$ denotes a basis of $K$ over $Q_{p}$, and $X \in I_{K}$ is assumed to be written in terms of this basis as

$$
X=x_{1} \Omega_{1}+\ldots+x_{N} \Omega_{N} \text {, where } x_{1}, \ldots, x_{N} \in I .
$$

However, we are in this paper not concerned with such general continuous functions, but with a subclass of continuous functions which have particularly simple properties. To this subclass one is led by an analogy from complex function theory.

An essential property of complex functions that are regular in a 
certain open set is that they are completely determined if their values are given on any arc in this set, or even only on an infinite sequence tending to a point in this set.

It seems therefore appropriate to consider in our p-adic case functions $F: I_{K} \rightarrow K$ which are continuous on $I_{K}$ and which are completely determined if their values on $I$ are given. Naturally, $F(X)$ is also continuous when $X$ is restricted to $I$, hence can on $I$ be written as the interpolation series

$$
F(X)=\sum_{n=0}^{\infty} A_{n}\left(\begin{array}{l}
X \\
n
\end{array}\right),
$$

where

$$
A_{n}=\sum_{k=0}^{n}(-1)^{k}\left(\begin{array}{l}
n \\
k
\end{array}\right) F(n-k) \quad(n=0,1,2, \ldots),
$$

where now the coefficients $A_{n}$ lie in $K$ and again form a null sequence.

DEFINITION. $F(X)$ is called a regular function on $I_{K}$ if the series (I) converges uniformly on $I_{K}$ and is identical with $F(X)$.

Our problem will be to find the basic properties of such regular functions and in particular their relation to analytic functions $G(X)$ on $I_{K}$. By this we mean the sums of power series

$$
G(X)=\sum_{n=0}^{\infty} G_{n} X^{n}
$$

which converge for all $X$ on $I_{K}$, hence which are characterised by the condition that $\left\{G_{n}\right\}$ is a null sequence. We shall find that in general the regular functions are identical with the analytic functions, but that there is an important special case when this is not so.

\section{1.}

Let $K$ be an algebraic extension field of degree $N$ over the rational p-adic field $Q_{p}$, and let $e$ and $f$ denote the ramification index and the residue class degree of $K$ over $Q_{p}$, respectively; hence 


$$
\text { ef }=N \text {. }
$$

As usual let $N(A)=N_{K / Q_{p}}(A)$ be the norm of $A$ in $K$.

The p-adic valuation $|a|_{p}$ of $Q_{p}$, which we assume normed such that

$$
|p|_{p}=p^{-1}
$$

has a unique continuation $|A|_{p}$ to $K$ defined by

$$
|A|_{p}=|N(A)|_{p}^{1 / N} \text { for } A \in K
$$

There exists then a prime element $P$ and a p-adic unit $E$ in $K$ such that

$$
p=E P^{e},|E|_{p}=1, \quad N(P)=p^{f},|P|_{p}=p^{-1 / e} .
$$

Denote by

$$
I=\left\{a \in Q_{p},|a|_{P} \leq I\right\} \text { and } I_{K}=\left\{A \in K,|A|_{P} \leq I\right\}
$$

the rings of all p-adic integers in $Q_{p}$ and of all $K$-integers in $K$; both sets are compact with respect to the metric induced by the p-adic valuation. Let further $R$ be a full set of $p^{f}$ representatives in $I_{K}$ of the different residue classes

$$
A(\bmod P)
$$

in $I_{K}$, where the class of the multiples of $P$ is represented by 0 . In the general case we may take for $R$ the set of all roots of the equation

$$
x^{p^{f}}-x=0
$$

in $I_{K}$. Further, in the special case when $e=N, f=1$, hence when $K$ is totally ramified, $R$ may be chosen as the set

$$
R=\{0,1,2, \ldots, p-1\} \text {. }
$$

With this definition of $R$, every $K$-integer has now the canonic representation 


$$
X=\sum_{h=0}^{\infty} x_{h} p^{h}
$$

where the coefficients $X_{h}$ are elements of $R$. On allowing also analogous terms in finitely many negative powers of $P$, we obtain a similar representation of the elements of $K$.

2.

Consider now any interpolation series (1) and assume that it represents a regular function $F(X)$ for all $X$ on $I_{K}$. It is not difficult to derive the necessary and sufficient conditions which the coefficients $A_{n}$ have then to satisfy.

For this purpose it is convenient to introduce the polynomials $(X, n)$ of $X$ which are defined by

$$
(X, n)= \begin{cases}1 & \text { if } n=0, \\ X(X-1) \ldots(X-n+1) & \text { if } n=1,2,3, \ldots,\end{cases}
$$

so that

$$
(X, n)=\left(\begin{array}{l}
X \\
n
\end{array}\right) \cdot n !
$$

To begin with, let

$$
1 \leq e<N, \quad 1<f \leq N,
$$

that is, $K$ is not totally ramified. There exists then an element $X_{0}$ of $R$ such that none of the numbers

$$
x_{0}-n \quad(n=0,1,2, \ldots)
$$

lies in the zero class $(\bmod P)$ and thus is divisible by $P$. Hence

$$
\left|\left(x_{0}, n\right)\right|_{p}=1 \quad(n=0,1,2, \ldots) .
$$

On the other hand, if $X$ is an arbitrary element of $I_{K}$, it is obvious that

$$
|(x, n)|_{p} \leq 1 \quad(n=0,1,2, \ldots)
$$


Now the series $F(X)$ is assumed to converge for all $X \in I_{K}$ and hence it converges in particular when $X=X_{0}$. But then the terms of the series $F\left(X_{0}\right)$ must tend to zero, and so it follows from (3) that necessarily

$$
\lim _{n \rightarrow \infty} \frac{A_{n}}{n !}=0
$$

Conversely, when this condition is satisfied, then, by (4), the series $F(X)$ converges uniformly for all $X \in I_{k}$ which implies that $F(X)$ is regular. Hence the following result has been proved.

THEOREM 1. If $K$ is not totally ramified, then $F(X)$ represents a regular function on $I_{K}$ if and only if its coefficients $A_{n}$ satisfy the limit condition (5).

3.

In the case (A) just considered, the condition (5) allows to connect the regular functions $F(X)$ to the analytic functions $G(X)$. For this purpose it suffices to note that, if $n \geq 1,(X, n)$ can be written as

$$
(x, n)=x^{n}+a_{n, 1} x^{n-1}+a_{n, 2} x^{n-2}+\ldots+a_{n, n-1} X,
$$

where the coefficients $a_{n, k}$ are rational integers, and that these equations can be solved for the different powers of $X$ in the form

$$
x^{n}=(x, n)+b_{n, 1}(x, n-1)+b_{n, 2}(x, n-2)+\ldots+b_{n, n-1}(x, 1),
$$

where also the coefficients $b_{n, k}$ are rational integers. It follows that the p-adic values $\left|a_{n, k}\right|_{p}$ and $\left|b_{n, k}\right|_{p}$ cannot be larger than 1 .

Therefore the two series

$$
F(X)=\sum_{n=0}^{\infty} A_{n}\left(\begin{array}{l}
X \\
n
\end{array}\right) \text { and } G(X)=\sum_{n=0}^{\infty} G_{n} X^{n}
$$

are formally identical exactly if

$$
A_{0}=G_{0}
$$


and if for $n=1,2,3, \ldots$ both

$$
\frac{A_{n}}{n !}=G_{n}+a_{n+1,1} G_{n+1}+a_{n+2,2} G_{n+2}+\ldots
$$

and

$$
G_{n}=\frac{A_{n}}{n !}+b_{n+1,1} \frac{A_{n+1}}{(n+1) !}+b_{n+2,2} \frac{A_{n+2}}{(n+2) !}+\ldots
$$

These two sets of formulae imply immediately the following result.

THEOREM 2. If formally $F(X)=G(X)$, then each of the two equations

$$
\lim _{n \rightarrow \infty} \frac{A_{n}}{n !}=0 \text { and } \lim _{n \rightarrow \infty} G_{n}=0
$$

implies the other one.

Since the first formula holds by Theorem 1 for regular functions and the second one is valid for analytic functions, we obtain then the following theorem.

THEOREM 3. If $K$ is not totally ramified, then the regular functions on $I_{K}$ are identical with the analytic functions on $I_{K}$. Hence such regular functions can be differentiated arbitrarily often, and all their derivatives are again regular functions on $I_{K}$.

For the $k$ th derivative

$$
G^{(k)}(x)=\sum_{n=k}^{\infty} n(n-1) \cdots(n-k+1) G_{n} x^{n-k}
$$

of $G(X)$ is likewise analytic on $I_{K}$ and so is also regular, and it formally is identical with $F^{(k)}(X)$.

$$
4 .
$$

There remains the case

$$
e=N, f=1
$$

when $K$ is totally ramified over $Q_{p}$. This case will prove to be more interesting and quite different from case (A). 
Now the general element $X$ of $I_{K}$ has the form

$$
X=\sum_{h=0}^{\infty} X_{h} P^{h},
$$

where the coefficients $X_{h}$ are restricted to the set

$$
R=\{0,1,2, \ldots, p-1\} \text {. }
$$

If the integer $n \geq 0$ satisfies the congruence

$$
n \equiv X_{0}(\bmod P) \text {, }
$$

then $X-n$ is divisible by at least the first power of $P$, and therefore

$$
|X-n|_{p} \leq|P|_{p}<1
$$

while otherwise

$$
|X-n|_{p}=1
$$

It follows that

$$
|(x, n)|_{p} \leq|P|_{p}^{[n / p]-1} \quad(n=0,1,2, \ldots)
$$

In the special case when $X=P$, this inequality can at once be replaced by the equation

$$
|(P, n)|_{p}=|P|_{p}^{[n / p]}
$$

For now $|P-n|_{p}$ is equal to 1 when $p$ does not divide $n$, but has the exact value $|P|_{p}$ when $p$ is a factor of $n$ because then $|n|_{p} \leq|p|_{p}<|p|_{p}$

Assume now that $F(X)$ is regular on $I_{K}$, thus that its interpolation series converges uniformly on this set. In particular, $F(P)$ must converge and so the terms of this series must tend to zero. Since, by (9),

$$
\left|\left(\begin{array}{l}
P \\
n
\end{array}\right)\right|_{p}=|n !|_{p}^{-1}|(P, n)|_{p}=|n !|_{p}^{-1}|P|^{[n / p]},
$$

this requires that 


$$
\lim _{n \rightarrow \infty} \frac{A_{n}}{n !} P^{[n / p]}=0 .
$$

Conversely, if this condition is satisfied, then for $x \in I_{K}$ the terms of the series for $F(X)$ tend by (8) uniformly to zero and hence the series converges uniformly. Thus we obtain the following result.

THEOREM 4. If $K$ is totally ramified, then $F(X)$ is a regular function on $I_{K}$ if and only if the coefficients $A_{n}$ satisfy the condition (10).

\section{5.}

Since $|P|_{p}=p^{-1 / N}<1$, the condition (10) is weaker than the condition (5) which had to be satisfied by analytic functions. It follows that in the case (B) regular functions need not be analytic. A simple example of such a regular non-analytic function is given by the series

$$
F(X)=\sum_{n=0}^{\infty} n ! P^{-[n / p]+[\log n]}\left(\begin{array}{l}
X \\
n
\end{array}\right)
$$

which obviously sttisfies the condition (10), but not the condition (5).

Since a regular function $F(X)$ in the case (B) is not in general analytic, it remains an open question whether it is differentiable or not on $I_{K}$. We shall now study this question in detail.

By the condition (10), the coefficients $A_{n}$ of $F(X)$ can be written as

$$
A_{n}=n ! P^{-[n / p]} \varepsilon_{n}(n=0,1,2, \ldots),
$$

where $\left\{\varepsilon_{n}\right\}$ is some null sequence in $I_{K}$. Let $Y$ be a second variable in $I_{K}$ and put

$$
B_{m n}=A_{m+n}\left(\begin{array}{l}
X \\
m
\end{array}\right)\left(\begin{array}{l}
Y \\
n
\end{array}\right) \quad(m, n=0,1,2, \ldots)
$$

By (11),

$$
B_{m n}=m ! P^{-[m / p]}\left(\begin{array}{l}
X \\
m
\end{array}\right) \cdot n ! P^{-[n / p]}\left(\begin{array}{l}
Y \\
n
\end{array}\right) \cdot \eta_{m n}(m, n=0,1,2, \ldots),
$$


where $\eta_{m n}$ denotes the number

$$
\eta_{m n}=\left(\begin{array}{c}
m+n \\
m
\end{array}\right) P^{[m / p]+[n / p]-[(m+n) / p]} \varepsilon_{m+n} \quad(m, n=0,1,2, \ldots) .
$$

Here, by (8),

$$
\left|m ! P^{-[m / p]}\left(\begin{array}{l}
X \\
m
\end{array}\right)\right|_{p} \leq|P|_{p}^{-1} \text { and }\left|n ! P^{-[n / p]}\left(\begin{array}{l}
Y \\
n
\end{array}\right)\right|_{p} \leq|P|_{p}^{-1}
$$

and it is also clear that

$$
\left|\left(\begin{array}{c}
m+n \\
m
\end{array}\right)\right|_{p} \leq 1 \text { and }[m / p]+[n / p]-[(m+n) / p] \geq-1 .
$$

Hence the first two factors of $B_{m m}$ are bounded for all $m$ and $n$, and the last factor $\eta_{m n}$ tends to zero when $m+n$ tends to infinity; the same is therefore true for $B_{m n}$ itself.

This proves that the double sum

$$
\Delta=\sum_{m=0}^{\infty} \sum_{n=0}^{\infty} B_{m n}
$$

converges uniformly for all $X$ and $Y$ in $I_{K}$. Since $K$ is nonarchimedean, we are allowed to order the terms of this double sum in any arbitrary order.

First take together all the terms of $\Delta$ for which $m+n$ has a fixed value $r$. Since

$$
\sum_{\substack{m+n=r \\
m \geq 0 \\
n \geq 0}}\left(\begin{array}{l}
X \\
m
\end{array}\right)\left(\begin{array}{l}
Y \\
n
\end{array}\right)=\left(\begin{array}{c}
X+Y \\
r
\end{array}\right),
$$

this gives

$$
\Delta=\sum_{r=0}^{\infty} A_{p}\left(\begin{array}{c}
X+Y \\
r
\end{array}\right)=F(X+Y) .
$$

Secondly, all the partial sums

$$
\sum_{m=0}^{\infty} B_{m}=\sum_{m=0}^{\infty} A_{m+n}\left(\begin{array}{l}
X \\
m
\end{array}\right)\left(\begin{array}{l}
Y \\
n
\end{array}\right)
$$


are convergent, and they tend to zero when $n$ tends to infinity. Put therefore

$$
A_{n}(X)=\sum_{m=0}^{\infty} A_{m+n}\left(\begin{array}{l}
X \\
m
\end{array}\right) \quad(n=0,1,2, \ldots) ;
$$

then

$$
\Delta=\sum_{n=0}^{\infty} A_{n}(X)\left(\begin{array}{l}
Y \\
n
\end{array}\right)
$$

and we obtain the identity

$$
F(X+Y)=\sum_{n=0}^{\infty} A_{n}(X)\left(\begin{array}{l}
Y \\
n
\end{array}\right) .
$$

Here, by our proof, the right-hand side converges uniformly in both variables.

6.

Before applying the last identity, let us investigate the sequence $\left\{A_{n}^{\prime}\right\}$ defined by the infinite series

$$
A_{n}^{\prime}=\sum_{m=1}^{\infty} \frac{(-1)^{m-1}}{m} A_{m+n} \quad(n=0,1,2, \ldots) .
$$

Here, by (11),

$$
\frac{1}{m} A_{m+n}=n ! P^{-[n / p]} \varepsilon_{m+n} \cdot\left(\begin{array}{c}
m+n \\
m
\end{array}\right)(m-1) ! P^{-[m / p]} \cdot P^{[m / p]+[n / p]-[(m+n) / p]} .
$$

We noted already that the p-adic value of $\left(\begin{array}{c}m+n \\ m\end{array}\right)$ is at most 1 and that $[m / p]+[n / p]-[(m+n) / p]$ is not less than -1 . Further, since

$$
|r !|_{p}=r^{-[r / p]-\left[r / p^{2}\right]-\cdots,}
$$

it is evident that both sequences

$$
\left\{(m-1) ! P^{-[m / p]}\right\} \text { and }\left\{n ! P^{-[n / p]}\right\} \text {, }
$$

where $m$ runs from 1 to infinity and $n$ from 0 to infinity, are bounded. It follows that there exists a positive constant $c$ independent of $m$ and $n$ such that 


$$
\left|\frac{1}{m} A_{m+n}\right|_{p} \leq c\left|n ! P^{-[n / p]} \varepsilon_{m+n}\right|_{p}
$$

for all suffices $m \geq 1$ and $n \geq 0$.

This property implies, firstly, that all the series (14) are convergent. Secondly, it follows from (14) and (15) that

$$
\left|A_{n}^{\prime}\right|_{p} \leq c\left|n ! p^{-[n / p]} \varepsilon_{n+1}\right|_{p}
$$

and hence that

$$
\lim _{n \rightarrow \infty} \frac{A_{n}^{\prime}}{n !} P^{[n / p]}=0
$$

This is, however, identical with the condition (10) for $A_{n}$. Therefore, by Theorem 4 , the new function

$$
F^{\prime}(X)=\sum_{n=0}^{\infty} A_{n}^{\prime}\left(\begin{array}{l}
X \\
n
\end{array}\right)
$$

defined for $X \in I_{K}$ is again regular. Our next question will concern the connection of $F^{\prime}(X)$ to the original regular function $F(X)$.

\section{7.}

By (8) and (15),

$$
\left|\frac{1}{m} A_{m+n}\left(\begin{array}{l}
X \\
n
\end{array}\right)\right|_{p} \leq c\left|n ! P^{-[n / p]} \varepsilon_{m+n} \cdot \frac{1}{n !} P^{[n / p]-1}\right|_{p}=c\left|P^{-1} \varepsilon_{m+n}\right|_{p} .
$$

This estimate proves immediately that the double series

$$
\nabla=\sum_{m=1}^{\infty} \sum_{n=0}^{\infty} \frac{(-1)^{m-1}}{m} A_{m+n}\left(\begin{array}{l}
X \\
n
\end{array}\right)
$$

converges uniformly for all $X \in I_{K}$. On summing first over $m$ and then over $n$, we find that

$$
\nabla=F^{\prime}(X)
$$

if, instead, we sum first over $n$ and then over $m$, the result takes the form 


$$
\nabla=\sum_{m=1}^{\infty} \frac{(-1)^{m-1}}{m} A_{m}(X)
$$

Hence $F^{\prime}(X)$ can be written as

$$
F^{\prime}(X)=\sum_{m=1}^{\infty} \frac{(-1)^{m-1}}{m} A_{m}(X)
$$

where $A_{m}(X)$ was defined by the formula (12). This equation shows already that $F^{\prime}(X)$ is closely related to $F(X)$.

To prove that the connection is even closer, we prove now that $F^{\prime}(X)$ is in fact the derivative of $F(X)$ at $X$, thus that

$$
F^{\prime}(X)=\lim _{Y \rightarrow 0} \frac{F(X+Y)-E(X)}{Y} .
$$

By the addition formula (13) and by the definition (12) of $A_{n}(X)$,

(18) $\frac{F(X+Y)-F(X)}{Y}=\frac{1}{Y} \sum_{n=1}^{\infty} A_{n}(X)\left(\begin{array}{l}Y \\ n\end{array}\right)=\sum_{n=1}^{\infty} \frac{A_{n}(X)}{n}\left(\begin{array}{c}Y-1 \\ n-1\end{array}\right)=$

$$
=\sum_{m=0}^{\infty} \sum_{n=1}^{\infty} \frac{A_{m+n}}{n}\left(\begin{array}{l}
X \\
m
\end{array}\right)\left(\begin{array}{l}
Y-1 \\
n-1
\end{array}\right) \text {. }
$$

A proof similar as before allows now to show that the double series

$$
\sum_{m=0}^{\infty} \sum_{n=1}^{\infty} \frac{A_{m+n}}{n}\left(\begin{array}{l}
X \\
m
\end{array}\right)\left(\begin{array}{c}
Y-1 \\
n-1
\end{array}\right)
$$

converges uniformly in $X$ and $Y$ when these run over $I_{K}$. For by (8) and $(11)$,

$$
\begin{aligned}
\left|\frac{A_{m+n}}{n}\left(\begin{array}{l}
X \\
m
\end{array}\right)\left(\begin{array}{l}
Y-1 \\
n-1
\end{array}\right)\right|_{p} & \leq \\
& \leq\left|\frac{(m+n) !}{n} P^{-[(m+n) / p]} \varepsilon_{m+n} \cdot \frac{1}{m !} P^{[m / p]-1} \cdot \frac{1}{(n-1) !} P^{[(n-1) / p]-1}\right|_{p}= \\
& \left.=\mid \begin{array}{c}
m+n \\
m
\end{array}\right) P \\
&
\end{aligned}
$$

because

$$
[(n-I) / p] \geq[n / p]-I \text { and therefore }[m / p]+[(n-1) / p]-[(m+n) / p] \geq-2 .
$$


It follows that the terms of the double series (19) tend to zero uniformly in $X$ and $Y$ as $m+n$ tends to infinity.

In order to determine the derivative of $F(X)$ at the point $X$ we are then allowed to let $Y$ tend to zero term by term on the right-hand side of (18), and then $\left(\begin{array}{l}Y-1 \\ n-1\end{array}\right)$ has the limit $(-1)^{n-1}$. It follows therefore from (17) and (18) that the function $F^{\prime}(X)$ already introduced is in fact the derivative of $F(X)$ at the point $X$.

This consideration gives therefore the following result.

THEOREM 5. If $K$ is totally ramified, then every regular function $F(X)$ on $I_{K}$ has a first derivative which is again a regular function. It has therefore regular derivatives of alz possible orders.

Since the regular function $F(X)$ is not in general analytic, the formal Taylor series

$$
\sum_{n=0}^{\infty} \frac{F^{(n)}(0)}{n !} x^{n}
$$

will not converge for all $X$ in $I_{K}$. It would have some interest to decide whether there always exists a positive integer $s$ such that this Taylor series converges for all $X$ satisfying $|X|_{p} \leq p^{-S}$. Another question left open is whether a regular function in the case (B) can vanish on an infinite subsequence of $I_{K}$ without being identically zero.

\section{Reference}

[1] K. Mahler, "An interpolation series for continuous functions of a p-adic variable", J. reine angew. Math. 199 (1958), 23-34.

Department of Mathematics, Institute of Advanced Studies, Australian National University, Canberra, ACT. 